\title{
NOTES
}

\section{Phylogenetic Position of Riemerella anatipestifer Based on 16S rRNA Gene Sequences}

\author{
SUMATHI SUBRAMANIAM, ${ }^{1,2}$ KIM-LEE CHUA, ${ }^{2}$ HAI-MENG TAN, ${ }^{2}$ HILDA LOH,${ }^{3}$ PETER KUHNERT, ${ }^{1}$ \\ AND JOACHIM FREY ${ }^{1 *}$
}

Institute for Veterinary Bacteriology, University of Bern, CH-3012 Bern, Switzerland ${ }^{1}$; Institute of Molecular Agrobiology, National University of Singapore, Singapore 05112; and Veterinary Laboratory Branch, Central Veterinary Laboratory, Singapore $1954^{3}$

\begin{abstract}
Riemerella anatipestifer, the causative agent of septicemia anserum exsudativa (also called new duckling disease), belongs to the family Flavobacteriaceae of gram-negative bacteria. We determined the DNA sequences of the rrs genes encoding the 16S rRNAs of four $R$. anatipestifer strains by directly sequencing PCR-amplified $r r$ genes. A sequence similarity analysis confirmed the phylogenetic position of $R$. anatipestifer in the family Flavobacteriaceae in rRNA superfamily $\mathrm{V}$ and allowed fine mapping of $R$. anatipestifer on a separate rRNA branch comprising the most closely related species, Bergeyella zoohelcum, as well as Chryseobacterium balustinum, Chryseobacterium indologenes, and Chryseobacterium gleum. The sequences of the rrs genes of the four $R$. anatipestifer strains varied between 0.5 and $1.0 \%$, but all of the strains occupied the same position on the phylogenetic tree. In general, differences in $r$ s genes were observed among $R$. anatipestifer strains, even within a given serotype, as shown by restriction fragment length polymorphism of PCR-amplified $r$ rs genes.
\end{abstract}

Riemerella anatipestifer is the etiological agent of septicemia anserum exsudativa, an enzootic, contagious, often primary septicemic disease of domesticated ducklings $(2,9)$. The disease has a worldwide distribution, and endemic infections are restricted to commercial duck and turkey flocks $(3,18)$. Mortality varies from 2 to $30 \%$, but it can be as high as $95 \%$ and is influenced by predisposing viral and bacterial infections. Thus, $R$. anatipestifer causes important losses in duck and turkey production units. $R$. anatipestifer is a gram-negative, nonmotile, non-spore-forming rod-shaped bacterium (3). So far, no particular virulence attributes have been described. Typing of $R$. anatipestifer isolates by slide and tube agglutination tests with antisera has identified 21 serotypes $(12,13)$.

The phylogenetic position of $R$. anatipestifer was uncertain for a long time. This organism was originally named Pfeiferella anatipestifer (9) and was subsequently named Pasteurella anatipestifer and Moraxella anatipestifer based more on host predilection than on conventional taxonomic criteria (5). Several phenotypic, morphological, and biochemical characteristics, including the lack of flagellation, a low DNA base ratio (29 to $35 \mathrm{~mol} \% \mathrm{G}+\mathrm{C}$ ), the presence of menaquinones as the sole respiratory quinones, the presence of branched-chain fatty acids at high levels, the absence of carbohydrate fermentation, and hydrolytic enzyme patterns, enabled classification of $R$. anatipestifer in the Flavobacterium-Cytophaga group (15), which was emended as the family Flavobacteriaceae $(4,19)$. Classification of $R$. anatipestifer in rRNA superfamily V, which contains the genera Chryseobacterium, Bergeyella, Ornithobacterium, Empedobacter, Weeksella, Capnocytophaga, Flavobacterium, Cytophaga, Flexibacter, and Sphingobacterium, was confirmed by DNA-DNA and DNA-rRNA hybridization analyses

\footnotetext{
* Corresponding author. Mailing address: Institute for Veterinary Bacteriology, Laenggassstrasse 122, CH-3012 Bern, Switzerland. Phone: 41-31-631 2484. Fax: 41-31-631 2634. E-mail: jfrey@vbi.unibe .ch.
}

$(3,4,17,19)$. We describe in this paper the DNA sequences of the entire 16S rRNA genes (rrs) from four $R$. anatipestifer strains. This information allowed us to determine precisely the phylogenetic position of $R$. anatipestifer in the Flavobacteriaceae rRNA homology group. Also, a comparison of the sequences revealed variations among $R$. anatipestifer strains.

Amplification of $16 S$ ribosomal DNA (rDNA). All of the strains used in this study are listed in Table 1 . The type strain is $R$. anatipestifer ATCC 11845. The serotype reference strains have been described previously (12). Field strains were isolated between 1983 and 1991 from diseased ducks and were typed by performing tube agglutination tests with antisera raised against $R$. anatipestifer serotype reference strains (12); these tests were performed by workers at the Primary Production Department, Central Veterinary Laboratory, Singapore. All of the strains were grown on Columbia agar plates at $37^{\circ} \mathrm{C}$ in air enriched with $5 \% \mathrm{CO}_{2}$ for $24 \mathrm{~h}$.

Genomic DNA was extracted by the rapid guanidium thiocyanate method of Pitcher et al. (14). PCRs were carried out in $50-\mu l$ reaction mixtures containing $5 \mu l$ of $10 \times$ PCR buffer supplemented with $\mathrm{MgSO}_{4}$ (Boehringer, Mannheim, Germany), 20 pmol of each primer, $1 \mathrm{mM}$ deoxynucleotide triphosphate (Boehringer), $2.5 \mathrm{U}$ of Pwo DNA polymerase (Boehringer), and $100 \mathrm{ng}$ of genomic DNA. PCR amplifications were performed with a GeneAmp PCR System model 2400 DNA thermal cycler (Perkin-Elmer) as follows: 35 cycles consisting of $94^{\circ} \mathrm{C}$ for $30 \mathrm{~s}, 54^{\circ} \mathrm{C}$ for $30 \mathrm{~s}$, and $72^{\circ} \mathrm{C}$ for $1 \mathrm{~min}$, with a final extension step consisting of $7 \mathrm{~min}$ at $72^{\circ} \mathrm{C}$ to allow all of the extension products to be completed. The primers used for PCR generation of $r r s$ gene fragments were universal primers 16SUNI-L and 16SUNI-R (11) derived from the Escherichia coli rrs sequence (6). Negative controls that contained all of the components described above except the template DNA were included in each experiment. The PCR products were examined by electrophoresis of small aliquots in a $0.7 \%$ agarose gel at $110 \mathrm{~V}$ for $2 \mathrm{~h}$. For further analysis the remainder of each PCR product was purified with a QIAquick PCR purification 
TABLE $1 . R$. anatipestifer strains

\begin{tabular}{|c|c|}
\hline Strain $^{a}$ & Serotype \\
\hline ATCC $11845^{\mathrm{T}}$. & $\mathrm{ND}^{b}$ \\
\hline $35 / 90 \ldots \ldots$. & 1 \\
\hline 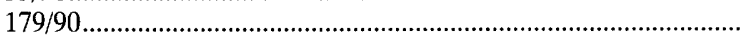 & 1 \\
\hline 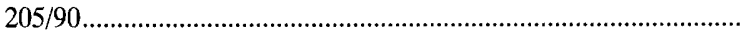 & 1 \\
\hline $105 / 91 \ldots$. & 1 \\
\hline 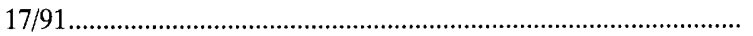 & 2 \\
\hline 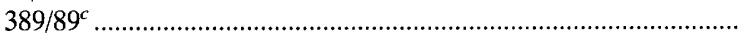 & 6 \\
\hline 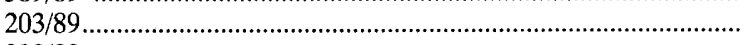 & 7 \\
\hline $232 / 89 \ldots .$. & - 10 \\
\hline$\ldots$ & 10 \\
\hline 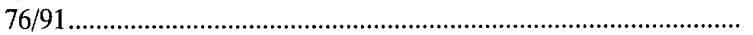 & 11 \\
\hline $84 / 91$ & 11 \\
\hline $134 / 90$ & 13 \\
\hline $664 / 83^{c}$ & 14 \\
\hline $743 / 85^{c}$ & 15 \\
\hline (1) & 15 \\
\hline $110 / 89$ & 15 \\
\hline $135 / 90$ & 15 \\
\hline $977 / 83^{c}$ & 17 \\
\hline 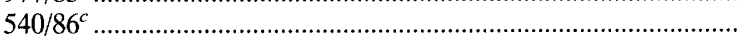 & 18 \\
\hline $30 / 90^{c} \ldots$. & 19 \\
\hline $53 / 91$ & 19 \\
\hline $59 / 91$ & 19 \\
\hline
\end{tabular}

${ }^{a}$ ATCC, American Type Culture Collection. All strains that were not type strains or serotype reference strains were field isolates of the Central Veterinary Laboratory, Singapore.

${ }^{b}$ ND, not determined.

${ }^{c}$ Serotype reference strain (12).

kit (Qiagen, Hilden, Germany) used according to the manufacturer's instructions.

Sequencing and phylogenetic analysis. Purified PCR amplification products of the 16S rRNA genes of type strain ATCC 11845 and the serotype 6,15 , and 19 reference strains were completely sequenced directly on both strands by using an AmpliTaq FS dye terminator kit (Perkin-Elmer) and oligonucleotide primers described previously (11). The primers were derived from the conserved regions of the 16S rRNA genes of many gram-negative and gram-positive bacterial species and were designed to allow complete sequencing of $r r s$ genes on both strands in one round. Each sequencing reaction was performed with approximately $50 \mathrm{ng}$ of template DNA per reaction mixture and $5 \mathrm{pmol}$ of primer. The sequences of the products were determined with an ABI Prism model 310 genetic analyzer and were edited and assembled by using the Sequencher 3.0 program (GeneCodes, Ann Arbor, Mich.) to obtain entire sequences. Alignment of the ATCC $11845^{\mathrm{T}}$ (T = type strain) $r r s$ gene sequence with EMBL and GenBank DNA and RNA sequence databank data by using the program BLASTN (1) revealed that the phylogenetically most closely related bacterial species all belonged to the Flavobacteriaceae rRNA homology group. Fourteen most closely related $16 \mathrm{~S}$ rRNA sequences and two more distantly related sequences were chosen for the phylogenetic analysis (Table 2). Each of the four $R$. anatipestifer rrs sequences was compared with the sequences of these 16 reference organisms. The evolutionary distances between sequences were computed by using the algorithm of Jukes and Cantor (10) with the Mega 1.02 phylogenetic analysis software package. Ambiguous nucleotides which were present in some sequences in the database were not considered in the comparison. A tree to estimate the phylogenetic relationships among the organisms was derived by using the neighbor-joining method (16) (Fig. 1). The topology of the distance tree was tested by performing 1,000 bootstrap
TABLE 2. Accession numbers of $16 \mathrm{~S}$ rRNA sequences of $R$. anatipestifer strains and related bacteria

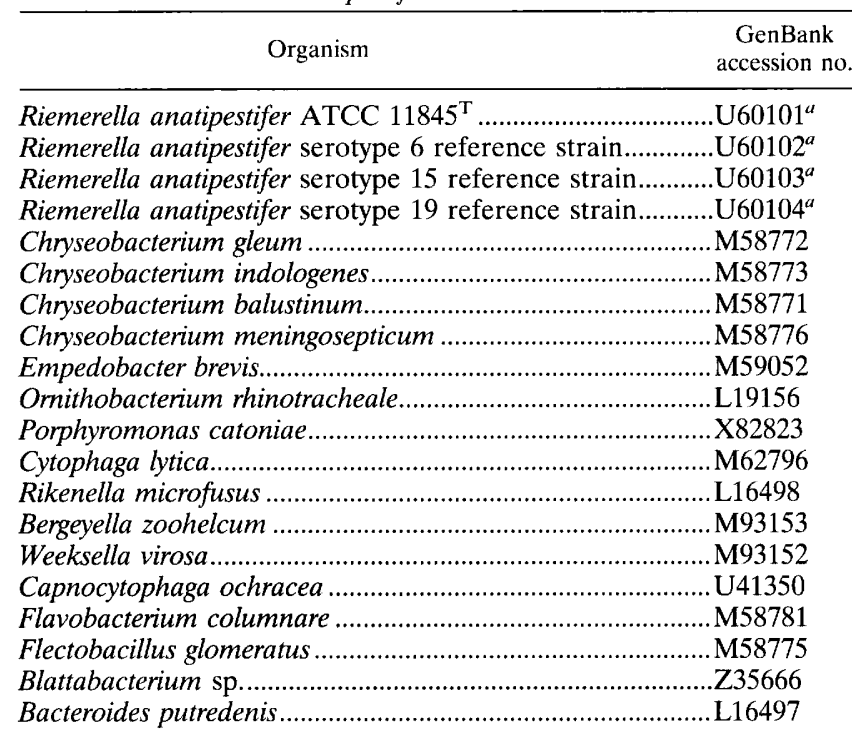

${ }^{a}$ Determined in this study.

resamplings of the data. Our analysis of the $16 \mathrm{~S}$ rRNA gene sequences of four different strains of $R$. anatipestifer confirmed the phylogenetic position of $R$. anatipestifer within rRNA superfamily $\mathrm{V}$ in the taxonomic neighborhood of the genera Chryseobacterium, Bergeyella, and Omithobacterium. The four $R$. anatipestifer strains occupied the same position on the phylogenetic tree (Fig. 1) generated in this study, which is branched like the tree based on DNA-rRNA hybridization analysis results (17). The organism that is most closely related to $R$. anatipestifer, Bergeyella zoohelcum, has $92.8 \%$ homolo-

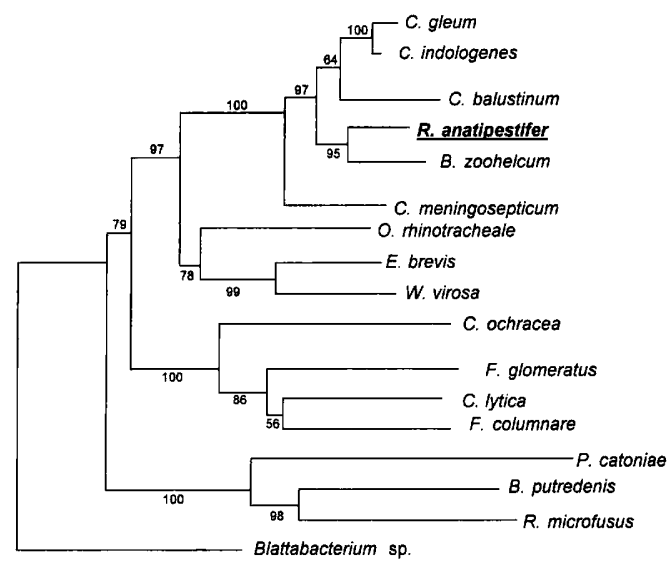

$\underset{0.01}{\longrightarrow}$

FIG. 1. Phylogenetic tree based on 16S rRNA gene sequence similarity values for $R$. anatipestifer type strain ATCC 11845 and 14 closely related species, as well as the more distantly related organisms Bacteroides putredenis and Blattabacterium sp. Phylogenetic relationships were established with the Mega 1.02 program; corrected distances were calculated with the Jukes-Cantor algorithm, and a tree was derived by using the neighbor-joining method. Bootstrap values were calculated from 1,000 trees. Scale bar $=1 \%$ sequence divergence. The $R$. anatipestifer reference strains for serotypes 6,15 , and 19 occupy the same phylogenetic position as the type strain despite the heterogeneity detected in the $16 \mathrm{~S}$ rRNA genes. See Table 2 for spelled-out names. 

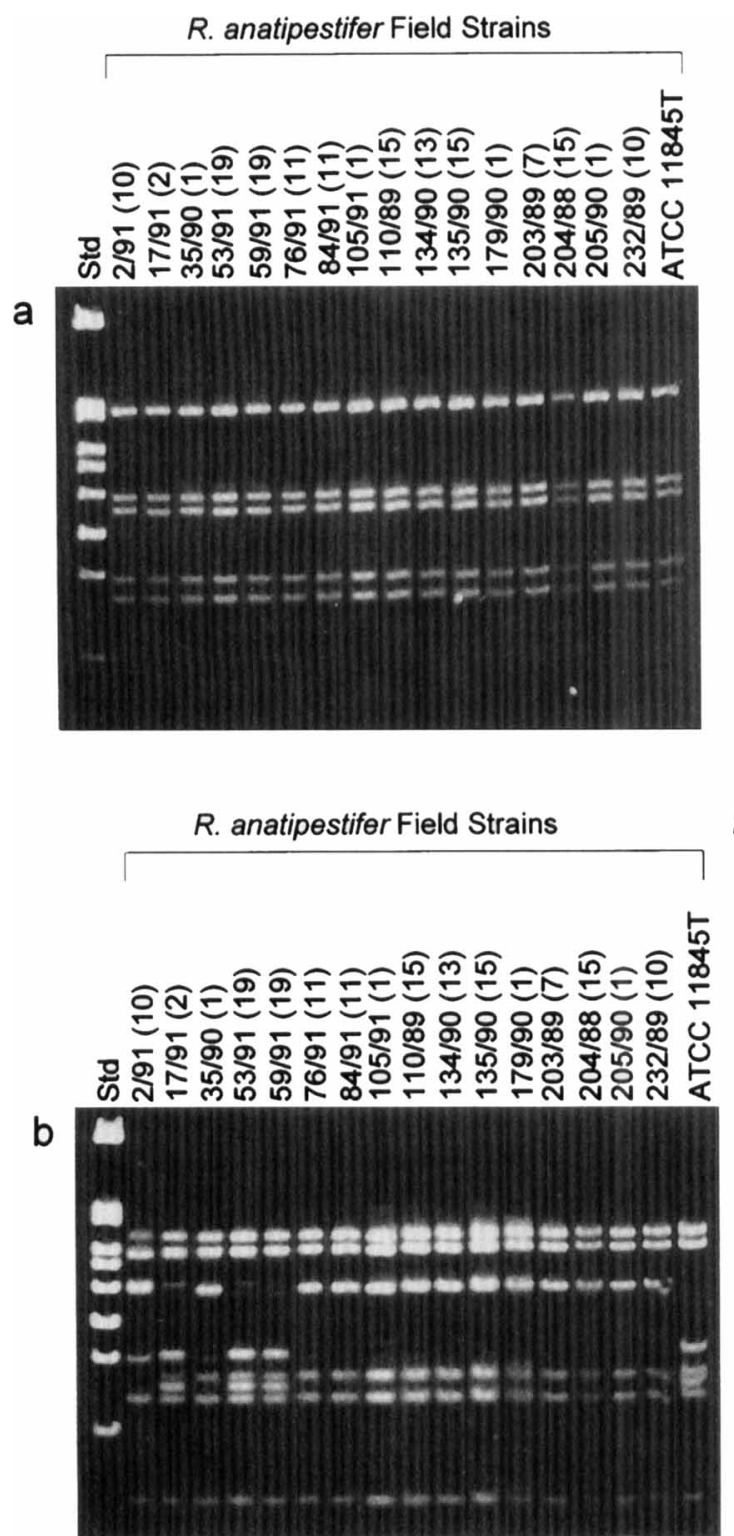

R. anatipestifer Reference Strains

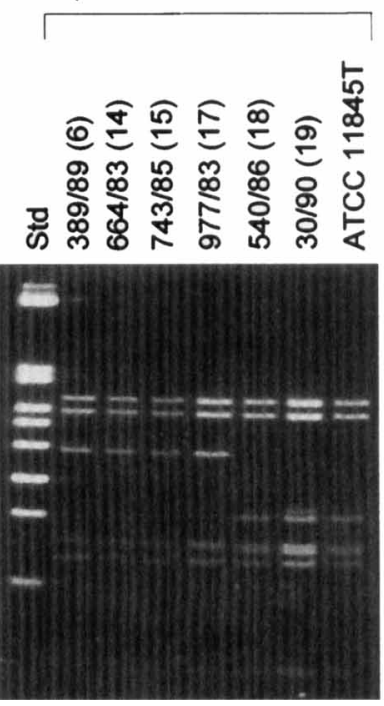

FIG. 2. RFLP analysis of $R$. anatipestifer strains. Chromosomal DNAs of serotype reference strains, the type strain, and field strains were extracted and used as templates for PCR amplification of 16S rRNA genes with universal primers. The PCR products were digested with enzymes, and the fragments were electrophoresed on $8 \%$ polyacrylamide gels. Bands were visualized by ethidium bromide staining. The standards used were Hinfl-digested pBR322 fragments. (a) HaeIII digests, showing identical RFLP patterns. (b) DdeI digests, showing minor heterogeneity in RFLP patterns among the strains. The numbers in parentheses indicate serotypes. Std, standard.

gous nucleotides in its $16 \mathrm{~S}$ rRNA sequence. Other closely related organisms are Chryseobacterium gleum, Chryseobacterium indologenes, Chryseobacterium balustinum, and Chryseobacterium meningosepticum, which exhibit 91.4 to $92.6 \%$ sequence homology in the $16 \mathrm{~S}$ rRNA genes. $R$. anatipestifer and $B$. zoohelcum each constitute a separate rRNA branch, while C. gleum, $C$. indologenes, and C. balustinum constitute another branch. Chryseobacterium meningosepticum occupies a separate branch.

Based on DNA-rRNA hybridization studies (17), $R$. anatipestifer was shown to belong to the family Flavobacteriaceae. $R$. anatipestifer, however, differs phenotypically from the genera Chryseobacterium and Bergeyella, which also belong to the Flavobacteriaceae rRNA homology group, by its capnophilic metabolism, by the absence of pigments, and by its fatty acid and protein contents (17). Our sequence data confirm the phylogenetic results obtained previously in DNA-rRNA hybridization studies (17). Moreover, our data allow fine-tuned classification of $R$. anatipestifer and affirm previous phenotypic and genotypic conclusions which support a separate genus for this taxon.

Alignment of the rrs sequences of the four $R$. anatipestifer strains examined (strain ATCC $11845^{\mathrm{T}}$ and the reference strains of serotypes 6,15 , and 19) revealed base differences at 14 positions. Of the 14 base differences, 3 (at positions 559, 747 , and 1190) were determined to be in conserved regions of the 16S rRNA gene of the family Flavobacteriaceae. Despite being in the conserved regions, the bases at two of the positions, positions 559 and 1190, seemed to be variable in members of the Flavobacteriaceae. 
RFLP. In order to obtain a broader view of the variability in the $r r s$ genes of $R$. anatipestifer, a restriction fragment length polymorphism (RFLP) analysis was performed with type strain ATCC 11845, serotype reference strains, and field isolates. Aliquots $(3 \mu \mathrm{l})$ of PCR products from the $R$. anatipestifer strains were digested with RsaI, AluI, HinfI, HaeIII, and DdeI. The products were examined on an $8 \%$ acrylamide gel electrophoresed with TBE buffer $(0.4 \mathrm{M}$ Tris, $0.4 \mathrm{M}$ boric acid, 0.01 $\mathrm{M}$ EDTA; $\mathrm{pH} 8.3$ ) for $4 \mathrm{~h}$ at $80 \mathrm{~V}$. The digested fragments were visualized by staining with ethidium bromide. The five enzymes were chosen on the basis of the rrs sequence of $R$. anatipestifer ATCC $11845^{\mathrm{T}}$.

The restriction fragment patterns obtained with $R s a \mathrm{I}-$, AluI-, HinfI-, and HaeIII-digested 16S rDNA PCR products were the same for all of the strains analyzed. The HaeIII restriction profiles are shown in Fig. 2a. Minor heterogeneity among the strains was observed in the restriction fragment profiles obtained with DdeI (Fig. 2b), which apparently divided the strains analyzed into two groups. This grouping of strains, however, seemed not to be useful. Strains 35/90 and 2/91, members of serotypes 1 and 10 , respectively, have $D d e$ I restriction profiles that are different from those of other strains belonging to these two serotypes (Fig. 2b). Therefore, DdeI profiles are not consistent with serotyping. In addition, the profiles were only dependent on a small number of significant bases in the $16 \mathrm{~S}$ rRNA gene (five restriction enzyme recognition sites, only two of which were located in the variable regions).

Alignments of $R$. anatipestifer rrs sequences revealed eight base differences between ATCC $11845^{\mathrm{T}}$ and serotype 19 and two base differences between serotypes 6 and 15, which have the same $D d e$ I restriction fragment profiles. Ten base differences were observed between ATCC $11845^{\mathrm{T}}$ and serotype 15 , which have different $D d e I$ restriction fragment profiles. This confirms that DdeI profiles do not distinguish different subtypes of $R$. anatipestifer.

Nucleotide differences in the $1 \%$ range were determined for the 16S rRNA genes of the four $R$. anatipestifer strains analyzed in this study. In addition, the results of the restriction fragment analysis of the $16 \mathrm{~S}$ rDNAs of $R$. anatipestifer strains revealed differences within a given serotype (Fig. 2b). These observations are not surprising as high levels of intraspecific variation (within and between strains) have been reported in the 16S rRNA sequences of prokaryotes in the GenBank database (7). It has been suggested that organisms with less than 15 base differences in the $16 \mathrm{~S}$ rRNA gene generally belong to the same species (8), a suggestion which seems to apply to $R$. anatipestifer.

The results obtained in this study are important for basic approaches to molecular analysis in $R$. anatipestifer since several functions and attributes are expected to vary among different strains, even within the same serotype. This should be particularly significant for future development of genetic methods for subtyping $R$. anatipestifer strains in order to avoid confusion due to subclassifications that are not congruent with serotypes.

Nucleotide sequence accession numbers. The sequence data are accessible under the following EMBL and GenBank accession numbers: ATCC $11845^{\mathrm{T}}$, U60101; serotype 6 reference strain 389/89, U60102; serotype 15 reference strain 743/85, U60103; and serotype 19 reference strain 30/90, U60104.
We thank J. Nicolet, Institute for Veterinary Bacteriology of the University of Bern, and N.-H. Chua, Laboratory of Plant Molecular Biology, Rockefeller University, New York, N.Y., for their support and encouragement. We also thank Y. Schlatter for her technical assistance.

This work was part of a Swiss Asia Foundation collaborative program between the Institute of Molecular Agrobiology of the National University of Singapore and the Institute for Veterinary Bacteriology of the University of Bern and was supported by grant 5002-038920 from the Priority Program Biotechnology of the Swiss National Foundation and the National Science and Technology Board, Singapore.

\section{REFERENCES}

1. Altshul, S. F., W. Gish, W. Miller, E. W. Myers, and D. J. Lipman. 1990 Basic local alignment search tool. J. Mol. Biol. 215:403-410.

2. Asplin, F. D. 1955. A septicemic disease of ducklings. Vet. Rec. 67:854-858. 3. Bangun, A., J. L. Johnson, and D. N. Tripathy. 1987. Taxonomy of Pasteu rella anatipestifer. I. DNA base composition and DNA-DNA hybridization analysis. Avian Dis. 31:43-45.

4. Bernardet, J.-F., P. Segers, M. Vancanneyt, F. Berthe, K. Kersters, and P. Vandamme. 1996. Cutting a Gordian knot: emended classification and description of the genus Flavobacterium, emended description of the family Flavobacteriaceae, and proposal of Flavobacterium hydatis nom. nov. (basonym, Cytophaga aquatilis Strohl and Tait 1978). Int. J. Syst. Bacteriol. 46:128-148.

5. Brogden, K. A. 1989. Pasteurella anatipestifer infection, p. 115-129. In C. Adlam and J. M. Rutter (ed.), Pasteurella and pasteurellosis. Academic Press, London, United Kingdom.

6. Carbon, P., C. Ehresmann, B. Ehresmann, and J. P. Ebel. 1979. The complete nucleotide sequence of the ribosomal 16-S RNA from Escherichia coli. Eur. J. Biochem. 100:399-410.

7. Clayton, R. A., G. Sutton, P. S. Hinkle, Jr., C. Bult, and C. Fields. 1995. Intraspecific variation in small subunit rRNA sequences in GenBank: why single sequences may not adequately represent prokaryotic taxa. Int. J. Syst. Bacteriol. 45:595-599.

8. Fox, G. E., J. D. Wisotzkey, and P. Jurtshuk, Jr. 1992. How close is close: 16S rRNA sequence identity may not be sufficient to guarantee species identity. Int. J. Syst. Bacteriol. 42:166-170.

9. Hendrickson, J. M., and K. F. Hilbert. 1932. A new serious septicemia disease of young ducks with a description of the new causative organism, Pfeifferella anatipestifer. Cornell Vet. 22:239-252.

10. Jukes, T. H., and C. R. Cantor. 1969. Evolution of protein molecules, p. 21-132. In H. N. Munro (ed.), Mammalian protein metabolism, vol. 3 Academic Press, Inc., New York, N.Y.

11. Kuhnert, P., S. E. Capaul, J. Nicolet, and J. Frey. 1996. Phylogenetic position of Clostridium chauvoei and Clostridium septicum based on 16S rRNA gene sequences. Int. J. Syst. Bacteriol. 46:1174-1176.

12. Loh, H., T. P. Teo, and H.-C. Tan. 1992. Serotypes of 'Pasteurella' anatipestifer isolates from ducks in Singapore: a proposal of new serotypes. Avian Pathol. 21:453-459.

13. Pathanasophon, P., T. Sawada, and T. Tanticharoenyos. 1995. New serotypes of Riemerella anatipestifer isolated from ducks in Thailand. Avian Pathol. 24:195-199.

14. Pitcher, D. G., N. A. Saunders, and R. J. Owen. 1989. Rapid extraction of bacterial genomic DNA with guanidium thiocyanate. Lett. Appl. Microbiol. 8:151-156.

15. Rossau, R. A., A. Van Landschoot, M. Gillis, and J. De Ley. 1991. Taxonomy of Moraxellaceae fam. nov., a new bacterial family to accommodate the genera Moraxella, Acinetobacter, and Psychrobacter and related organisms. Int. J. Syst. Bacteriol. 41:310-319.

16. Saitou, N., and M. Nei. 1987 . The neighbor-joining method: a new method for reconstructing phylogenetic trees. Mol. Biol. Evol. 4:406-425.

17. Segers, P., W. Mannheim, M. Vancanneyt, K. De Brandt, K.-H. Hinz, K. Kersters, and P. Vandamme. 1993. Riemerella anatipestifer gen. nov., comb. nov., the causative agent of septicemia anserum exsudativa and its phylogenetic affiliation within Flavobacterium-Cytophaga rRNA homology group. Int. J. Syst. Bacteriol. 43:768-776.

18. Singh, R., M. F. Teng, T. P. Teo, and E. K. Kua. 1983. Anatipestifer disease in ducklings in Singapore. Singapore Vet. J. 7:53-57.

19. Vandamme, P., J.-F. Bernardet, P. Segers, K. Kersters, and B. Holmes. 1994 New perspectives in the classification of the flavobacteria: description of Chryseobacterium gen. nov, and Empedobacter nom. rev. Int. J. Syst. Bacteriol. 44:827-831. 